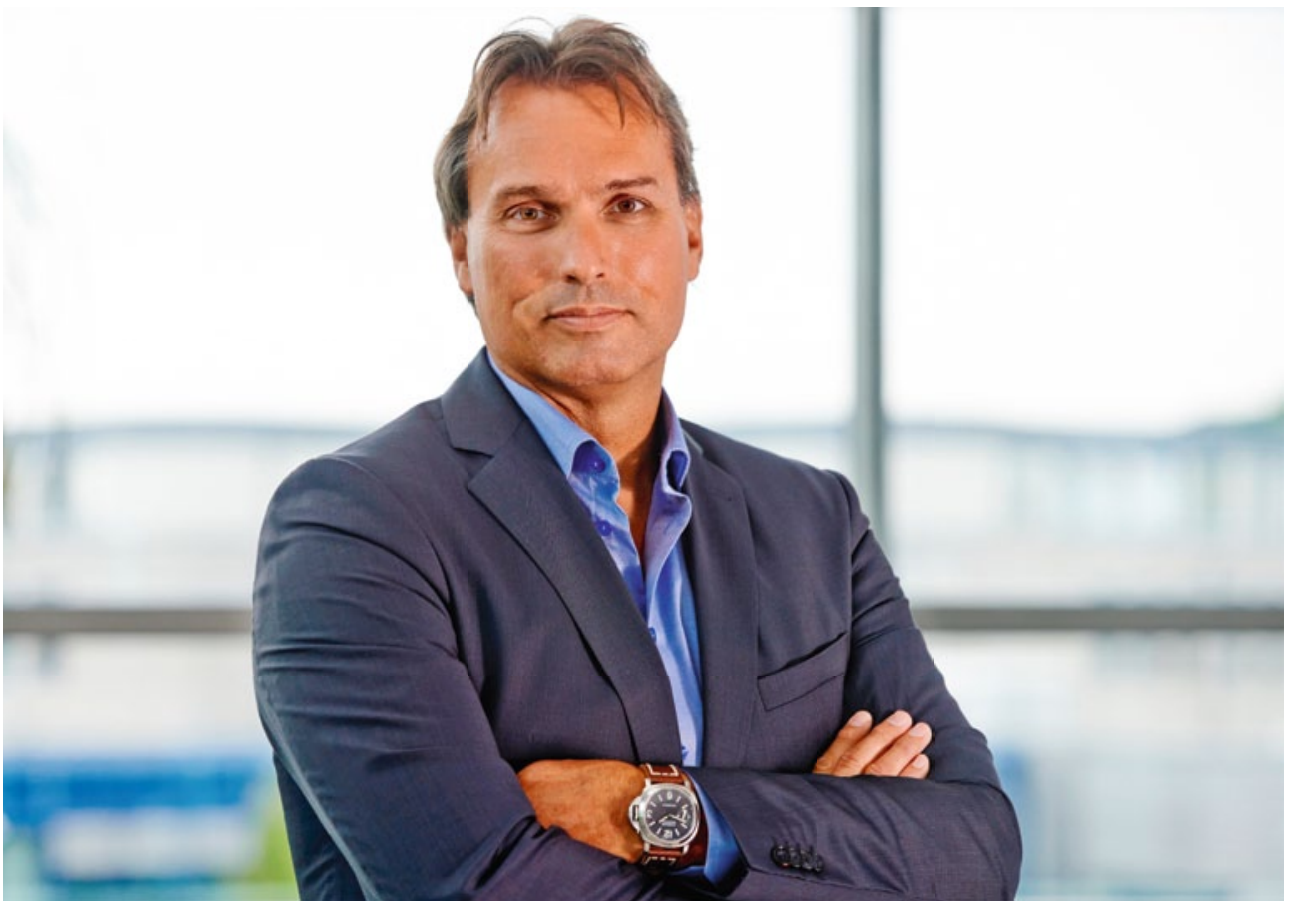

PROF. DR.-ING. PIM VAN DER JAGT

Executive Technical Leader, Vehicle Dynamics Research \& Advanced Engineering, Ford-Forschungszentrum Aachen $\mathrm{GmbH}$

\title{
DAS VERNETZTE AUTO DER ZUKUNFT
}

Technische Meilensteine hat es in den letzten Jahrzehnten so einige gegeben: Sicherheitsgurt, Katalysator, ABS, Airbag, ESP, die Lithium-Ionen-Batterie und vieles mehr. Für erhöhte Sicherheit, mehr Effizienz und bessseren Komfort reiht sich nun die Kommunikation des Fahrzeugs mit anderen Autos oder der Infrastruktur in diese Auflistung ein.

$\mathrm{Zu}$ den in Feldversuchen getesteten Car-to-X-Technologien gehören sowohl reine Warnsysteme als auch Systeme, die noch einen Schritt weitergehen und im Notfall eingreifen. Ein Beispiel, wie intelligente Fahrzeuge den Straßenverkehr sicherer gestalten können, ist das „elektronische Notbremslicht“, das Ford im Rahmen des Verbundprojekts SimTD entwickelt hat. Diese Funktion unterstützt den Fahrer im Fall von stark bremsenden Fahrzeugen vor ihm, die beispielsweise hinter Kurven nicht sichtbar sind, und kann helfen, Auffahrunfälle zu vermeiden. Bremst der Fahrer eines Fahrzeugs sehr stark, wird dieses als Ereignis erkannt und zusammen mit Fahrzeugdaten wie Position, Geschwindigkeit und Fahrtrichtung an andere Fahrzeuge übertragen. Nachfolgende Fahrzeuge empfangen diese Nachricht und warnen den Fahrer.

Die Datenübertragung erfolgt entweder über W-Lan oder über Mobilfunk. Letzteres hat den Nachteil, dass beim Datentransfer sicherheitsrelevanter Informationen beziehungsweise Eingriffe nicht garantiert werden kann, dass es nicht zu inakzeptablen Zeitverzögerungen kommt. Für die Datenübertragung per W-Lan wiederum muss erst die entsprechende ins
Fahrzeug integrierte Infrastruktur geschaffen werden. Zur Umsetzung der Fahrzeug-zu-X-Kommunikation wurden spezielle Kommunikationsprotokolle entwickelt und standardisiert, beispielsweise IEEE 802.11p und ETSI ITS G5. Um die Einführung kooperativer Systeme vorzubereiten, ist es essenziell wie bei kaum einem anderen Thema, dass sich Automobilhersteller, Zulieferer, Forschungsinstitute und Regierungen zusammenschließen und an diversen Forschungsprojekten weltweit beteiligen. Über die eigentliche Technik hinaus müssen natürlich vor allem Fragen der Datensicherheit sowie der gesetzlichen Rahmenbedingungen adressiert und geklärt werden.

Da die Nutzung der Fahrzeug-zu-X-Kommunikation zur Steigerung der Effizienz nicht ganz so zeitkritisch ist, ist eine frühere Einführung mittels mobilfunkbasierter Informationsübertragung denkbar. Hierbei lässt sich der Verkehrsfluss dadurch erhöhen, dass die Fahrer kontinuierlich aktuelle Informationen zu Staus oder Baustellen erhalten. Eine der größten Herausforderungen bei der Einführung der Car-to-X-Kommunikation wird es sein, die anfängliche „Durststrecke“ zu überwinden. Denn ihre wahre Stärke spielt diese Technik erst mit wachsender Marktdurchdringung aus, das heißt, je mehr Fahrzeuge damit ausgestattet sind, desto größer wird der Einfluss auf die Verkehrssicherheit und -effizienz. In der Phase mit geringer Marktpenetranz könnte das Angebot an zu empfangenen Informationen seitens der Infrastruktur erheblich dazu beitragen, dass Kunden sich trotzdem für ein solches System entscheiden. 